\title{
Margot Jefferys: a voz britânica da sociologia médica
}

\author{
Margot Jefferys: the British voice of medical sociology
}

Everardo Duarte Nunes ${ }^{1}$

\footnotetext{
${ }^{1}$ Departamento de Medicina Preventiva e Social, Faculdade de Ciências Médicas, Universidade Estadual de Campinas. Rua Tessália Vieira de Camargo 126, sala 27. 13083-970 Distrito de Barão Geraldo Campinas SP.

evernunes@uolcom.br
}

\begin{abstract}
Margot Jefferys (1916-1999) was not only the person who introduced medical sociology into Great Britain, but also the researcher and professor who, during thirty years, exerted the most deep influence on the teaching of health social sciences either in undergraduate or especially graduate studies, since the beginning of her career, in 1953, at the London School of Hygiene and Tropical Medicine. In addition to create a global panorama concerning the Jefferys' works, this study highlights two texts, which are situated into the field we are researching: the history of health sociology. The first one, published in 1991, discusses the relationships between epidemiology and sociology; and the second, published in 1996, discusses the field of medical sociology. Both texts are a series of considerations regarding the Jeffer$y$ s' thought and the more recent questions of the field in Great Britain.
\end{abstract}

Key words Margot Jefferys, Medical/health sociology, History, Social sciences in medical schools, Medical/Great Britain sociology
Resumo Margot Jefferys (1916-1999) não foi apenas a pessoa que introduziu a sociologia médica na Grã-Bretanha; foi a pesquisadora e professora que durante trinta anos exerceu a mais profunda influência no ensino das ciências sociais em saúde, tanto na graduação como especialmente na pós-graduação, desde o início da sua carreira, em 1953, na London School of Hygiene and Tropical Medicine. Neste estudo, além de um panorama geral sobre os trabalhos de Jefferys, são destacados dois textos que se situam no campo que estamos pesquisando: a história da sociologia da saúde. O primeiro trata das relações entre a epidemiologia e a sociologia, publicado em 1991, e o segundo, publicado em 1996, é sobre o campo da sociologia médica. São tecidas considerações sobre o pensamento de Jefferys e as questões mais recentes do campo na Grã-Bretanha.

Palavras-chave Margot Jefferys, Sociologia médica/saúde, História, Ciências sociais nas escolas médicas, Sociologia médica/Grã-Bretanha 
Moreover, there has been a serendipity in the specific area in which I have worked. I did not consciously seek or prepare myself for work in the sociology of medicine; it was a series of chance factors which brought me into it.

However, I have never for one moment found the sociological issues that are posed by health related activities and institutions dull or intellectually unrewarding. On the contrary, they have offered and will continue to offer many exciting challenges. ${ }^{1}$

\section{Introdução}

Margot Jefferys (1916-1999) ${ }^{1}$ não foi apenas a pessoa que introduziu a sociologia médica na Grã-Bretanha, foi a pesquisadora e professora que durante trinta anos exerceu a mais profunda influência no ensino das ciências sociais em saúde, tanto na graduação como especialmente na pós-graduação, desde o início da sua carreira, em 1953, na London School of Hygiene and Tropical Medicine. Fui seu aluno nos anos 70, no curso Sociology applied to medicine, e pude testemunhar a sua capacidade de liderança marcada pela balizada formação em economia e ciência política, assim como conhecer o seu passado de militância política durante a Segunda Guerra. Neste estudo, além de um panorama geral sobre os trabalhos de Jefferys, são destacados dois textos que se situam no campo que estamos pesquisando: a história da sociologia da saúde. O primeiro trata das relações entre a saúde pública/epidemiologia e a sociologia ${ }^{2}$, que a autora retomaria, analisando a sua própria inserção no campo da saúde ${ }^{3}$, e o segundo, publicado em 1996, que Margot Jefferys havia apresentado como conferência em $1994^{4}$.

\section{Perfil biográfico}

Margot Davies nasceu em Madras (Índia), em $1^{\circ}$ de novembro de 1916, e foi para a Inglaterra com oito anos para ser educada na Berkhamsted School, in Hertfordshire, tradicional escola inglesa fundada em 1541. Bacharel em história econômica, em 1938, pela London School of Economics, a sua carreira acadêmica foi marcada pelas seguintes posições: Lecturer, Department of $\mathrm{Pu}$ blic Health, London School of Hygiene and Tropical Medicine 1953-65, Director of Social Research, Bedford College, London University 196582, Senior Lecturer in Sociology 1965-66, Reader
1966-68, Professor of Medical Sociology 1968-82 (Emeritus).

Meg Stacey (1922-2004), figura proeminente da sociologia médica e do movimento feminista britânico, lembra algumas das suas marcantes características pessoais: Uma profunda crença na igualdade, liberdade e justiça guiou sua vida, na política e na academia. Os eventos dos anos $1930-$ desemprego em massa, marchas de famintos, a guerra civil espanhola, a perseguição nazista aos judeus e oponentes políticos - levou-a, como a muitos outros intelectuais, ao Partido Comunista. Neste contexto, ela, com James Jefferys, com quem havia se casado em 1941 [a separação do casal ocorreu em 1959], tornou-se uma operária em fábricas de munições em Coventry ${ }^{5}$, cidade e área metropolitana na região denominada West Midlands, Inglaterra, que sofreu graves ataques aéreos dos alemães na Segunda Guerra. Margot relata em sua autobiografia: Eu despendi muitos anos da guerra trabalhando em uma fábrica como operadora de torno mecânico durante o dia e ensinando, à noite, história do trabalho ou assuntos do momento para grupos da Worker's Education Association formados por mineiros e engenheiros ${ }^{1}$. Diz que o trabalho na fábrica era extremamente cansativo, pelo caráter rotinizado que tinha, mas, por outro lado, proporcionava o contato direto com as experiências do ordinary worker ${ }^{1}$.

Margot permaneceu no Partido Comunista até 1956, quando os tanques soviéticos invadiram a Hungria. Embora tendo abandonado o Partido, não deixou de militar na new left e de lutar pelos direitos políticos de muitas pessoas. Como exemplo, cite-se a correspondência enviada ao British Medical Journal, em 1976, sobre o "desaparecimento" de dois médicos chilenos, Ivan Inzunza e Carlos Godoy, vítimas da repressão. Nessa carta, escreve que os dois médicos haviam trabalhado no governo de Salvador Allende e estavam "desaparecidos", mas que As autoridades chilenas dizem que elas nada sabem sobre eles, mas este é um método que usaram no passado para prevenir futuros inquéritos ${ }^{6}$. Autora de uma obra reconhecida internacionalmente, Margot recebeu em 1979 o Leo G. Reeder Award pela sua notável contribuição à sociologia médica. Faleceu em Londres, no dia 3 de março de 1999.

\section{O legado de Margot Jefferys}

As suas pesquisas iniciais foram sobre mobilidade no mercado de trabalho (Mobility in the labour market ${ }^{7}$ ) e serviços sociais (An anatomy of 
Social Welfare services ${ }^{8}$ ). Os biógrafos de Jefferys assinalam que durante esse período ela juntouse a vários cientistas sociais para discutir temas relacionados às relações entre classe social e saúde. Dentre eles destacam-se Ann Cartwright e Raymond Illsley. Cartwright (1925) teve papel destacado nos estudos sobre a prática médica na Inglaterra, associando às suas pesquisas as análises estatísticas; Illsley, abordando os mais diferentes assuntos, em especial sobre as relações saúde e desigualdades sociais. Lembre-se de que Raymond Illsley foi o primeiro diretor do Aberdeen MRC Medical Sociology Unit, tendo permanecido na cidade de 1965 a 1985.

A grande influência exercida por Jefferys desenvolve-se quando ela assume a docência no Bedford College da Universidade de Londres, no curso de pós-graduação em sociologia médica, reconhecido internacionalmente. O Bedford College é hoje parte integrante do Royal Holloway and Bedford New College da University of London.

O seu papel na introdução das ciências sociais nos cursos de graduação de medicina ocorreu após o impacto provocado por The Todd Report ${ }^{9}$, elaborado pela Royal Commission on Medical Education, a partir de 1963, e das suas críticas a esse relatório. Embora o relatório tenha recomendado um substancial aumento no ensino de psicologia e sociologia para os estudantes de medicina e o urgente desenvolvimento da medicina social e de comunidade ${ }^{10}$, Jefferys sugere que esse documento reafirmou a idéia de que a ciência social não tinha papel no treinamento de médicos, quando recomendava que qualquer contribuição da sociologia para a educação médica devia ser feita pelos cientistas do comportamento empregados nos departamentos da universidade. A Comissão sugeria que se cientistas sociais fossem empregados pelas escolas médicas, perderiam sua identidade intelectual com a sua disciplina de origem. Jefferys não concorda com esta posição, acreditando que se a questão fosse ganhar legitimação, então aqueles que ensinam essa disciplina deviam estar imersos no empreendimento médico ${ }^{11}$. Jefferys mostra que essa história mudou ao longo dos anos, e hoje as escolas médicas empregam seus próprios cientistas sociais, sendo ela uma das principais responsáveis pelo avanço do ensino das ciências sociais em diversos níveis.

Se a educação médica foi uma das frentes de atuação ${ }^{12}$, certamente a sua presença nos assuntos sobre os serviços de saúde e a prática médica, em especial a prática do médico generalista, foi uma constante. Em 1970, Jefferys liderou o grupo que elaborou o Relatório da British Medical
Association Working Group on Primary Health Care, "talvez o mais independente dos relatórios de planejamento da BMA”. Data de 1971 a publicação do trabalho em que foram pesquisados 776 representantes da prática geral na Grã-Bretanha. Nesse estudo, os médicos que trabalhavam em centros de saúde são comparados com os profissionais em outras práticas de grupo e com médicos que trabalham sozinhos. Dentre os achados da pesquisa, destacou-se o fato de que os participantes dos centros de saúde são os médicos mais jovens, que a proporção de médicos que não trabalha em grupos estava diminuindo rapidamente e que esses médicos são mais idosos $^{13}$. Muitos aspectos administrativos e de organização são discutidos, e este trabalho com dados do final dos anos 60 mostra a importância crescente dada à avaliação contínua do National Health Service. Outros trabalhos sobre a prática do generalista aparecem nos anos 70 .

Nos anos 80, muitos seriam os seus trabalhos tratando do serviço de saúde inglês ${ }^{14-17}$. Juntamente com o grupo de trabalho sobre cuidado primário, que organizou, a partir de 1983 publicou Rethinking General Practice: dilemmas in primary medical care, com a coautoria de Hessie Sachs ${ }^{18}$, e em 1988 recebeu o Honorary Fellowsihp do Royal College of General Practitioners. Data do final dos anos 80 a sua análise sobre as perspectivas do National Health Service para os anos 90, e dez anos depois, em 1998, novamente, as suas contribuições seriam fundamentais para a elaboração do livro considerado a versão histórica mais completa do Serviço Nacional de Saúde da Grã-Bretanha, desde a sua criação em 1948 até 1997, organizado por Loudon et al. ${ }^{19}$.

O tema do envelhecimento seria também umas das suas tarefas ao longo dos anos $80 \mathrm{e}$ década de 90. Assim, publicou, no final dos anos 80, a coletânea Growing old in the twentieth century ${ }^{20}$, que havia sido antecedida por um trabalho sobre o futuro do envelhecimento na GrãBretanha $^{21}$. Em 1991, juntamente com dois outros pesquisadores, entrevistou 73 "pioneiros da medicina geriátrica”, em um projeto de pesquisa de histórias de vida denominado The development of geriatric specialty. Essas entrevistas foram transcritas e depositadas na British Library Sound Archive para futuras pesquisas. Comentando este projeto, Bornat ${ }^{22}$ assinala que o interesse era "assegurar um lugar na história para uma especialidade [a geriatria] marginalizada”.

Outros temas que iriam acompanhar a trajetória de Jefferys voltam-se para a medicina soci$\mathrm{al}^{3,23,24}$ e ciências sociais ${ }^{25-27}$, além de uma diversi- 
dade de assuntos como eutanásia ${ }^{28}$, menopau$\mathrm{sa}^{29,30}$, consumo de medicamentos ${ }^{31}$ e outros. Pesquisadora atenta, muitas são as resenhas que elaborou e publicou nos principais periódicos da área da saúde.

\section{Relações interdisciplinares: sociologia/saúde pública}

Quando escreveu o artigo que trata das relações entre a sociologia médica e a saúde pública, em particular com a epidemiologia ${ }^{2}$, a situação do ensino das ciências sociais no Reino Unido era diferente daquela do final dos anos 60. Novas recomendações haviam sido feitas pelos comitês de educação médica, e como escreve a autora, Espera-se, hoje, que todos os estudantes de medicina e odontologia no Reino Unido tenham algum conhecimento rudimentar de sociologia e de sua contribuição potencial para a teoria e prática da medicina. São também introduzidos nos princípios da epidemiologia e estatística médica, e seu papel na pesquisa e na prática medicas ${ }^{2}$. Diz ainda que há restrições e escassos recursos para essas disciplinas, mas poucos negariam o seu valor e sua acreditação junto ao ensino, acrescentando que embora as forças dominantes nos estabelecimentos médicos não fossem idênticas, apresentavam consideráveis similaridades ${ }^{2}$ em aceitar essas disciplinas. Como analisa, os praticantes de saúde pública, embora vistos pelos médicos clínicos como aberrant physicians, vinham da tradição do treinamento médico, ao passo que os sociólogos médicos eram outsiders in the medical world. A entrada dos cientistas sociais no mundo acadêmico, no Reino Unido, foi por meio da psiquiatria e da saúde pública, pelo fato de esses dois campos, respectivamente, reconhecerem a importância dos determinantes sociais no comportamento e considerarem que as análises das desigualdades sociais necessitavam dos fundamentoss teóricos da epidemiologia e da medicina social e não da bacteriologia e da química inorgânica ${ }^{2}$. Mas, como lembra Jefferys ${ }^{2}$, se essas áreas ofereceram "abrigo", não foi "puramente por razões altruístas"; ante o prestígio das outras especialidades, tanto entre o público como entre os estudantes, a psiquiatria e a saúde pública passam a ser vistas com certas simpatias, na medida em que aumenta a prevalência das doenças mentais e o arcabouço da medicina somática não parece dar conta dessa situação, e na medida em que a organização dos serviços de saúde passam por reformulações.
Nesse texto, a autora trata de uma questão que iria acompanhar as discussões da sociologia médica, no sentido de situá-la quer seja no interior da prática médica (sociologia na medicina), quer seja a partir dos marcos teóricos da sociologia (sociologia da medicina), e não auxiliar um grupo profissional dominante para exercitar seu poder mais efetivamente $^{2}$. A essas críticas juntaram-se nos anos 70 e 80 as vozes feministas contra uma sociologia médica, que acusavam os sociólogos médicos de ajudar a perpetuar a dominação masculina sobre as experiências de saúde das mulheres ${ }^{2}$.

A importância deste trabalho está no fato de apontar para questões que continuam desafiando o campo da saúde coletiva - as tensões entre os proponentes (e propostas) da sociologia e da epidemiologia e que no Reino Unido estão associadas à estrutura organizacional dos serviços de saúde, incluindo estabelecimentos educacionais. Para Jefferys ${ }^{2}$, embora o sistema de saúde britânico tenha passado por um processo de liberalização, as "mensagens" da epidemiologia e da sociologia têm sido aceitas de forma "parcial e relutante". No entanto, assinala que as tensões têm um lado positivo; não se trata de um "choque" entre as disciplinas. Para a autora, na média, o epidemiologista está apto em concentrar-se em uma extensão mais estreita de problemas e fatores que o envolvem e valorizar a escolha sobretudo de métodos estatísticos como instrumentos analíti$\cos ^{2}$. Comparativamente, a média dos sociólogos é tão consciente do contexto social mais amplo no qual os problemas existem, que estão insatisfeitos com investigações estatísticas parciais, que falham em fornecer explicações satisfatórias da influência das estruturas e processos sociais sobre as formas individuais e coletivas do comportamento humano ${ }^{2}$. Encerra chamando a atenção para o fato de que as tensões sobre "o discurso intelectual referente às teorias e métodos” podem fortalecer o campo de ideias na saúde.

\section{A sociologia médica na teoria e na prática}

O artigo que será analisado intitula-se "The development of medical sociology in theory and practice in Westen Europe 1950-1990" ". Quando apresentou este trabalho, Margot já havia percorrido o longo caminho que ela, junto com um grupo de pesquisadores, havia feito no sentido de construir e institucionalizar o campo da sociologia médica na Inglaterra. Assim, embora outros trabalhos sobre a Inglaterra e países europeus tenham sido elaborados, a pertinência de suas observações e a 
forma de contextualizar a sociologia médica/saúde permanecem como instigadoras mais de uma década após a sua publicação.

A autora divide o texto em duas partes. $\mathrm{Na}$ primeira, percorre cinco décadas da organização dos serviços de saúde relacionando-as com os avanços nas ciências médicas e mudanças nos padrões econômicos, sociais e culturais da sociedade como um todo. Jefferys alia essas transformações aos interesses teóricos e de práticas políticas dos cientistas sociais. Retomando alguns pontos deste texto, verificamos que, ao trabalhar o contexto sociomédico, lembra que o interesse sociológico sobre a saúde dos indivíduos não era um fenômeno inteiramente novo nos anos 90 . O estudo de Durkheim sobre o suicídio e diversos trabalhos demográficos e dos reformadores ingleses do século XIX tinham evidenciado os efeitos das privações sociais nos diferenciais de mortalidade das distintas classes sociais. Em realidade, as teorias clássicas marxistas, weberianas e durkheiminianas deram pouca atenção às questões da saúde, e mesmo as abordagens parsonianas dos anos 50 demoraram a chegar aos países europeus. Jefferys ressalta que, na Europa, "a resposta inicial a essas ideias foi um tanto abafada" 4 . Situa que foi nos anos 60, ou um pouco antes, que os sociólogos na Europa Ocidental voltaram-se para os estudos das relações entre as funções de controle social dos sistemas de cuidados de saúde e a autoridade dos médicos e das instituições hospitalares onde trabalhavam. Recorda a influência das abordagens marxistas, inicialmente, e depois as inspiradas pelas perspectivas foucaultianas. Assinala que $A o$ mesmo tempo - pelo menos na Grã-Bretanha - os cientistas sociais com backgrounds ecléticos criados em abordagens pragmáticas de temas sociais e informados por uma mistura de disciplinas das ciências sociais, incluindo teorias do campo político e social e da psicologia social, foram voltando cada vez mais a atenção para a politica social do Welfare State ${ }^{4}$. Os exemplos que se destacam nessa direção referem-se às relações indivíduo/Estado e às consequências da intervenção do Estado na economia de mercado, assim como às relações entre empregadores e empregados na indústria e entre profissionais e clientes na provisão de serviços. Ao explicar o porquê desses acontecimentos, assinala que A turbulência nas sociedades ocidentais como um todo e em suas partes constituintes inevitavelmente afetou as preocupações daqueles que tentaram usar a disciplina intelectual da sociologia para entender melhor os processos sociais do mundo contemporâneo, incluindo aqueles gerados no cuidado da saúde ${ }^{4}$. Nesse momento, a autora levanta um ponto importante em relação aos cientistas sociais que se dedicaram não somente a conhecer a realidade do mundo, mas tentaram usar os instrumentos e conhecimentos analíticos de suas disciplinas para ajudar a alterar os balanços de poder, em favor daqueles considerados em desvantagens e/ou privados em relação àqueles que, convencionalmente, são dotados com os poderes de status e posição $o^{4}$. Cita como um exemplo importante de manifestação contra a ordem social existente dentro da saúde que tem suas raízes nos anos 60 e começou a exercer grande influência sobre os padrões profissionais e de comportamento do paciente ${ }^{4}$ o novo movimento feminista. Para Jefferys, a saúde e o cuidado à saúde assumem outra escala de importância e passam a ser objeto de interesse dos sociólogos teóricos, analistas de políticas sociais e ativistas radicais a fim de implementar a mudança nas principais instituições da sociedade. Diante dessa situação, não é estranho que "houvesse muito pouca coerência na emergente sociologia médica" ${ }^{4}$. Nesse ponto de seu trabalho, a autora volta-se para a questão que já havia sido assinalada por Straus - a sociologia "da" e "na" medicina, assinalando o caráter teórico eclético da sociologia "da" medicina, convivendo com uma sociologia "na" medicina de caráter pragmático, sem que esta, muitas vezes, assuma as proposições de uma sociologia "da" medicina. Esta constitui a segunda parte do seu trabalho: as relações simbióticas entre as duas sociologias. Assume que se trata de uma análise a partir da perspectiva britânica e da sua experiência pessoal. Explica que, no período que cobre a sua análise, as relações entre os acadêmicos e os que pretendiam planejar ou "operar" no cuidado à saúde eram problemáticas. Se, de um lado, os sociólogos "da" medicina ampliam a sua abordagem renomeando o campo como "sociologia da saúde", de outro os sociólogos "na" medicina aceitam que as bases de suas intervenções repousam nos achados das pesquisas que "lançam suas luzes" sobre as problemáticas levantadas pelas práticas das organizações de saúde dos países, que por sua vez se situam no campo maior do "superestado", i.e., a União Europeia, e das próprias profissões de saúde ${ }^{4}$. Esclarece Jefferys ${ }^{4}$ que, durante o período, os adeptos de cada abordagem distinguiram-se não apenas pelo foco, mas pelas bases institucionais: os sociólogos "da" medicina nos departamentos de sociologia e os sociólogos "na" medicina nos departamentos das escolas médicas ou nos estabelecimentos associados às práticas clínicas e de saúde pública. 
Esse trabalho teve bastante repercussão; dentre os comentários, selecionei o de Denny Vage$\mathrm{ro}^{32}$, que havia cursado o Bedford College em 1979 e tinha sido aluno de Margot Jefferys, Utta Gerhard e George Brown. Nesse texto, Vagero recupera a importância que teve o curso de ciências sociais aplicadas à medicina, lembrando que um expressivo número de pesquisadores seniores na Europa (eu acrescento: e de outros países) formaram-se nesse curso. Lembra o comentarista que o ceticismo dos sociólogos da medicina frente os sociólogos na medicina, elaborado por Margot Jefferys, é talvez mais pronunciado na Grã-Bretanha do que em outros lugares. Nos países escandinavos, por exemplo, a maioria dos sociólogos médicos provavelmente tem uma orientação dirigida para a saúde pública. Para algumas pessoas seriam definidos como trabalhando em sociologia na medicina, mas eles não são menos independentes, em estabelecer a sua própria agenda de pesquisa, do que aqueles sociólogos que estudam a organização do cuidado médico ou o relacionamento médico-paci$e^{e n t e} e^{32}$. Acredita que a sociologia médica deve conservar um pé em ambos os campos. Chama ainda a atenção para a especificidade que o campo da sociologia médica apresenta em relação às características de transição sociopolítica que alguns países (naquele momento) atravessavam, tema não abordado por Jefferys em seu estudo, embora ela tenha enfatizado a importância do contexto sociopolítico e do contexto da saúde no qual cresceu a sociologia médica ${ }^{32}$.

\section{Comentários finais}

No início deste trabalho, foi situada a ampla gama de interesses de Jefferys no campo da sociologia médica/saúde. A diversidade temática abordada por Jefferys reflete a progressiva ampliação do campo da sociologia na saúde ao largo dos anos e pode ser vista no levantamento realizado e apresentado neste trabalho.

Retomam-se nestes comentários finais dois pontos que são as marcas deixadas por Jefferys para os estudos posteriores na área da sociologia da saúde, além da sua forte presença no ensino superior de graduação em medicina e na pós-graduação no campo das ciências sociais da saúde. Trata-se dos estudos sobre o envelhecimento e da análise dos sistemas de saúde. Nesse sentido, o testemunho de Mike Bury é extremamente interessante. Bury ${ }^{33}$ relata de forma detalhada a importância do trabalho e do convívio com Margot Jefferys quando torna-se lecturer no Bedford
College, nos anos 80, partindo para estudos sobre doenças crônicas, a interrupção das atividades por motivo de doença e a volta, no momento em que Margot estava se aposentando, e como os seus interesses de pesquisa tornaram-se convergentes. Como escreve, esses interesses focalizaramse particularmente sobre o envelhecimento e a gerontologia social e que Meu próprio trabalho sobre doença crônica e incapacidade tinha frequentemente tocado temas referentes ao envelhecimento, mas eu não tinha focalizado-os suficientmente como poderia ter feito ${ }^{33}$. Bury lembra que nos anos 80 vai se firmando a ideia de uma "sociedade de idosos" e que Margot Jefferys teve papel fundamental em chamar a atenção para as transformações de uma sociedade que envelhecia, fato que hoje é totalmente evidente. Bury, atualmente professor emérito da University of London, iria produzir muitos trabalhos e, sem dúvida, com a influência recebida de Margot Jefferys, que prefaciou seu livro sobre a velhice, de $1991^{34}$.

Em relação às questões sobre os sistemas de saúde, este foi, como apresentado neste trabalho, um dos temas favoritos de Jefferys - inclusive constituía a sua parte no curso de pós-graduação quando ministrava a disciplina de sistemas comparativos de saúde. Sem dúvida, a contribuição que permanece até os dias atuais, fruto do seu trabalho, é a perspectiva sociopolítica que imprimiu às suas análises ao longo da sua vida profissional, incluindo os seus últimos trabalhos. Rosemary Stevens ${ }^{35}$, ao resenhar os principais trabalhos escritos quando o National Health Service completou cinquenta anos, em 1998, ressaltou o trabalho de Margot Jefferys.

Peça importante para se entender a trajetória do campo, em seus anos iniciais, e a posição assumida por Margot Jefferys é a sua autobiografia, que escreveu em 1978 e que faz parte do livro que apresenta as histórias de vida de muitos sociólogos da medicina, como diz o título at work. Ao relatar a sua formação, lembra que na London School of Economics, onde se graduou, a sua escolha foi em ciências sociais em um departamento que buscava uma abordagem desenvolvimentista da sociedade em lugar da exegese das contribuições feitas pelas diferentes escolas de pensamento sociológico para uma compreensão das estruturas sociais contemporâneas ${ }^{1}$. Voltando-se para a área que constituiria a sua escolha profissional, o estudo dos sistemas de saúde, acrescenta: A probabilidade de eu ver o serviço de saúde contemporâneo como o produto de um longo desenvolvimento moldado por diferentes fatores em vez de um sistema de transações no qual os atores 
individuais negociam os resultados a serem obti$d o s^{1}$. Esta seria a visão dominante em seus trabalhos: a tendência em adotar uma perspectiva macroscópica e não microscópica teria sido, como declara, reforçada pela ausência de qualquer introdução à psicologia social, sem desvalorizar aqueles que escolhem usar conceitos sociológicos tais como poder, autoridade, controle, rótulo e estigma para explicar a natureza das relações e comportamentos dos pequenos grupos como a família ou as situações diádicas face a face tais como as relações médico-pacientes ${ }^{1}$.

Certamente, a sociologia médica britânica não se limitou às temáticas e aos enfoques dados por Margot Jefferys e, progressivamente, incorporou assuntos que se tornariam dos mais importantes nas pesquisas realizadas pelos pesquisadores, especialmente nos anos 80 e 90 do século passado.

Como visto no texto de Jefferys $(1996)^{4}$ e comentado por Annandale e Field ${ }^{36}$, a agenda dos 70 estava carregada de um "médico-centrismo", ou seja, a tendência em tomar como referência o que a medicina considerava problemático e digno de estudo. Não se pode esquecer que foi nos anos 70 que a sociologia médica tornou-se parte dos cursos de graduação médica no Reino Unido, tendo expressivo avanço no período de 1978 a $1998^{36}$. Em relação às questões atuais da sociologia médica na Grã-Bretanha, ressaltam os autores que o centro das preocupações dos cientistas está em investigar as relações da medicina/serviço de saúde com a sociologia, que têm sido marcadas, nos anos 90, pela natureza extremamente aplicada de muitos projetos de pesquisa e falta de atenção para os temas sociológicos mais amplos ${ }^{36}$. São citadas como focos de atenção algumas áreas: análises da experiência com a saúde e a doença, desigualdades sociais (relacionadas à classe social e, mais recentemente, ao gênero, "raça" e idade) e a provisão de cuidado à saúde (formal e informal). Do ponto de vista teórico, desponta a partir de 1994 o conceito de "risco", na perspectiva das ciências sociais. Também verificam-se novos focos empíricos de interesse, tais como os que se voltam, nos anos 90, para a ênfase sobre o life course, por exemplo, nas pesquisas sobre o desenvolvimento da saúde da criança.

Sem dúvida, a liderança de Margot Jefferys, que se estendeu por várias décadas, seu papel no campo da sociologia médica e suas atividades no campo educacional trazem a marca do pioneirismo que se destaca pelo espírito crítico e reflexivo.

Ao realizarem abalizada revisão do campo, Annandale e Field ${ }^{36}$ expressam que No princípio do século XXI, como nos anos 60, os sociólogos médicos britânicos permanecem fortemente influenciados pelas demandas algumas vezes competitivas da pesquisa aplicada induzidas pelas agendas politicas e o desejo de contribuir para o desenvolvimento de conceptualizações sociológicas mais adequadas da saúde e da doença. Não discordamos dos autores, mas acreditamos que o grande esforço de Margot Jefferys foi o de tentar aliar os desenvolvimentos dentro da sociologia como uma disciplina e os interesses práticos da política de saúde, como apontam Annandale e Field ${ }^{36}$. 


\section{Referências}

1. Jefferys M. Serendipity: an autobiographical account of the career of a medical sociologist in Britain. In: Elling RH, Sokolowska M, editors. Medical sociologists at work. New Brunswick: Transaction Books; 1978. p. 135-162.

2. Jefferys M. Medical sociology and public health: interdisciplinary relationships 1950-90. Public Health 1991; 105(1):15-21.

3. Jefferys M. Social Medicine and Medical Sociology 1950-1970: the testimony of a partisan participant. In: Porter D, editor. Social Medicine and Medical Sociology in the twentieth century. Amsterdam/Atlanta, GA: Rodopi B. V.; 1997. p. 120-136.

4. Jefferys M. The development of medical sociology in theory and practice in Western Europe 1950-1990. The European Journal of Public Health 1996; 6(2):9498.

5. Stacey M. Obituary. The Independent (London) 1999; 12 mar.

6. Jefferys M. Plight of Chilean doctors. Br Med J 1976; 4; 2(6035):583.

7. Jefferys M. Mobility in the labour market: employment changes in Battersea and Dagenham. London: Routledge Kegan Paul; 1954.

8. Jefferys M. An anatomy of Social Welfare services. London: Michael Joseph; 1965.

9. Lord Todd (Chairman). Report of the Royal Commission on Medical Education 1965-68. London: HMSO; 1968.

10. Draper P, Grenholm G, Best G. The organization of health care: a critical view of the 1974 reorganization of National Health Service. In: Tuckett D, editor. An introduction to Medical Sociology. London: Tavistock Publications; 1976. p. 254-290.

11. Porter D. Introduction. In: Porter D, editor. Social Medicine and Medical Sociology in the twentieth century. Amsterdam/Atlanta, GA: Rodopi B. V.; 1997. p. 131 .

12. Jefferys M, Elston MA. The medical school as a social organization. Med Educ 1989; 23(3):242-251.

13. Irvine D, Jeffreys M. B.M.A. Planning Unit Survey of General Practice 1969. Br Med J 1971; 27; 4(5786):535540 .

14. Jefferys M, Sachs H. Re-thinking general practice: some issues of policy and practice in the 1970s illustrated by case study. Soc Hist Med Bul 1983; 33:17-19.

15. Jefferys M. Britain's National Health Service under pressure. J Med Pract Manage 1985; 1(2):147-152.

16. Jefferys M. Britain's National Health Service in 1986: comments from a native user. $J$ Med Pract Manage 1986; 2(2):135-140.

17. Jefferys M. Britain's National Health Service: into the 1990s. J Med Pract Manage 1989; 5(2):91-95.

18. Jefferys M, Sachs H. Rethinking general practice: dilemmas in Primary Care. London: Tavistock; 1983.

19. Loudon I, Horder J, Webster C. General practice under the National Health Service 1948-1997. Oxford: Oxford University Press; 1998.
20. Jefferys M, editor. Growing old in the twentieth century. London: Routledge; 1989.

21. Jefferys M. An ageing Britain: what is its future? Geriatr Nurs Home Care 1987; 7(7):19-24.

22. Bornat J. Recycling the evidence: different approaches to the reanalysis of gerontological data. Forum: Qualitative Social Research [on-line journal] 2005; 6(1). [acessado 2007 out 13]. Disponível em: http:// www.qualitative-research.net/fqs-texte/1-05/05-1-42e.htm

23. Jefferys M. The boundaries of community medicine with the social work services. Public Health 1969; 84(1):29-45.

24. Jefferys M. The transition from public health to community medicine: the evolution and execution of a policy for occupational transformation. Bulletin of the Society for the Social History of Medicine 1986; 39:47-63.

25. Jefferys M. Sociology in medicine. Med World 1963; 98:239-240.

26. Jefferys M. Sociology and medicine separation or symbiosis? Lancet 1969; 1(7606):1112-1116.

27. Jefferys M. Social science and medical education in Britain: a sociologic analysis of their relationship. Int J Health Serv 1974; 4(3):549-563.

28. Howarth G, Jefferys M. Euthanasia: sociological perspectives. Br Med Bull 1996; 52(2):376-385.

29. McKinlay S, Jefferys M, Thompson B. An investigation of the age at menopause. J Biosoc Sci 1972; 4(2):161-173.

30. McKinlay SM, Jefferys M. The menopausal syndrome. Br J Prev Soc Med 1974; 28(2):108-115.

31. Jefferys M, Brotherston JH, Cartwright A. Consumption of medicines on a working-class housing estate. Br J Prev Soc Med 1960; 114:64-76.

32. Vagero D. European medical sociology: a comment on Margot Jefferys' view. European Journal of Public Health 1996; 6(2):98-99.

33. Bury M. In sickness and in health: working in medical sociology. In: Oliver A, editor. Personal histories in health research. London: The Nuffield Trust; 2005.

34. Bury M, Holme A. Life after ninety. London: Routledge; 1991.

35. Stevens RA. Fifty years of the British National Health Service: mixed messages, diverse interpretations. Bulletin of the History of Medicine 2000; 74(4):806811.

36. Annandale E, Field D. Medical sociology in Great Britain. In: Cockerham WC, editor. The Blackwell companion to Medical Sociology. Oxford, UK: Blackwell Publishers; 2001. p. 246-262.

Artigo apresentado em 25/02/2008

Aprovado em 28/03/2008

Versão final apresentada em 10/04/2008 